\title{
INTERPLAY OF COMMUNICATION AND COMPUTATION ENERGY CONSUMPTION FOR LOW POWER SENSOR NETWORK DESIGN
}

\author{
Zeeshan Ali Khan, Mustafa Shakir \\ Department of Electrical Engineering, \\ COMSATS Institute of Information Technology, \\ Islamabad, Pakistan. \\ \{zakhan, mustafa.shakir\} @comsats.edu.pk
}

\begin{abstract}
The sensor network design approach normally considers the communication energy consumption for evaluating a communication protocol. This is true for the low power devices such as MICAz/MICA2 which do not consume a lot of energy for the data treatment. However, recently developed sensor devices for multimedia applications such as iMote 2 do consume considerable amount of energy for data processing. In this article, we consider various scenarios for routing the data in wireless multimedia sensor networks by considering the local design parameters of devices such as PXA27x and beagleboard. The proposed routing solution considers node level optimizations such as data compression, dynamic voltage and frequency scaling (DVFS) for making a routing decision. The proposed approaches have been simulated to prove the effectiveness of the approach.
\end{abstract}

\section{KEYWORDS}

Wireless Sensor Networks, Low Power Design, Communication versus Computation Energy Consumption, Real-time data delivery.

\section{INTRODUCTION}

The wireless sensor networks are employed in various monitoring applications. The main optimization aspect of the sensor network is to minimize the energy consumption by choosing an energy efficient data dissemination strategy. These routing strategies normally consider the communication energy consumption and ignore the computation energy consumption. This is true for sensor devices such as MICA2/MICAz/TelosB as they normally have a micro controller that operates at the lower frequency which minimizes the computation energy consumption.

However, the multimedia sensor network applications have to do a lot of computation before sending the data on the wireless networks. Thus, in this case we cannot ignore this energy consumption while making a decision at the routing layer. The strategies that significantly effect the computation energy consumption include the video compression in the multimedia transmission that consumes a considerable amount of energy.

The article is organized as follows; section 2 describes the context of the presented work with respect to the related work in the field of energy efficient routing and node level optimizations in wireless sensor networks. Then, the chosen platform and proposed routing solution is explained in sections 3 and 4. Finally, the experimental results are explained in section 5. 


\section{CONTEXT}

A large volume of sensor network research is focussed on applications where the processor is only capable of dealing with low data rate applications such as temperature and pressure monitoring. However, there is a new paradigm in research where there are sensor network applications which have to process a large amount of data. Here, the existing techniques can not be directly applied [1]. In general, the sensor networks are deployed in regions where it is difficult to replace the battery. Thus, it is necessary to have power efficient algorithms at the system as well as network layer. In this case, we need to consider the local optimizations such as data compression, dynamic voltage and frequency scaling (DVFS) and dynamic power management (DPM) while making a routing decision. Thus, we need to find an interrelation between node level and network level design techniques in order to optimize the overall design. There are some techniques where authors have developed the power consumption model of a specific device and used it to make the energy efficient algorithms [2].

There is a lot of work that has been done using DVFS in the wireless sensor networks. These include [3] which propose an energy efficient task scheduling for structural health monitoring by considering dynamic voltage and frequency scaling. [4] explains the design of a low power video monitoring application on wireless sensor network using 802.15.4 protocol. They also explain the power consumption strategy by using the DVFS strategy at the system level.

Now we describe how this type of routing can be used inside a wireless sensor network deployed using imote 2 or TelosB. The energy consumption profile for these nodes is listed in table 1 for different data compression techniques such as lossless, lossy and raw technique. The raw technique sends the data without any compression, lossless compression ratio reduces the size of data by 3 and lossy compression reduces the size of data 10 times. For raw data transmission, the energy consumption on imote 2 platform is $1242 \mathrm{~J}$ while this energy consumption is $374.4 \mathrm{~J}$ on the TelosB platform. The energy consumption for the lossless compression is $835.5 \mathrm{~J}$ for imote 2 platform while it is $4618 \mathrm{~J}$ for the TelosB platform. This shows that local optimizations such as compression can significantly reduce the device's overall energy consumption by reducing the size of transmitted data. This energy consumption is computed for the network diagram shown in figure 1 . The results are listed in table 2 and it shows that iMote 2 has higher energy consumption for the transmission, but we can efficiently compress the data using this platform to decrease the global energy consumption.

In this article, we shall discuss first the local optimizations which are possible and then we shall explain their relation with the network layer design.

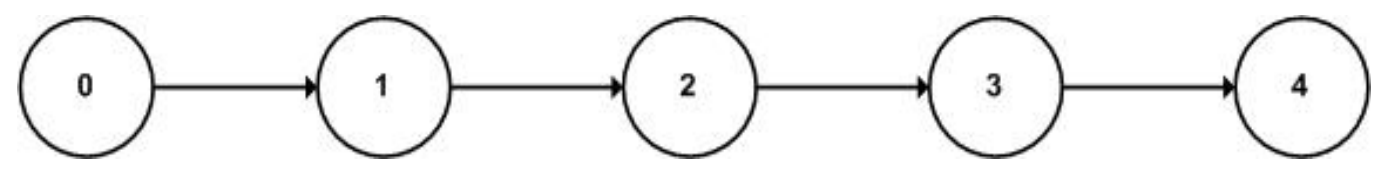

Figure 1. Example Network 
Table 1. Compression Energy Consumption for TelosB versus iMote2 [1]

\begin{tabular}{|c|c|c|c|c|c|c|}
\hline & \multicolumn{3}{|c|}{ Imote2 (J) } & \multicolumn{3}{c|}{ Telos (J) } \\
\hline & Computation & Transmission & Total & Computation & Transmission & Total \\
\hline Raw & 0 & 1242 & 1242 & 0 & 374.4 & 374.4 \\
\hline Lossless & 7.7 & 827.7 & 835.5 & 4369 & 250 & 4618 \\
\hline Lossy & 12.3 & 124.1 & 136.4 & 6480 & 37.4 & 65.7 \\
\hline
\end{tabular}

Table 2. Analytical Evaluation on Example Network (Figure 1)

\begin{tabular}{|c|c|c|c|c|}
\hline & \multicolumn{2}{|c|}{ Imote2 (J) } & \multicolumn{2}{c|}{ Telos (J) } \\
\hline & $\begin{array}{c}\text { Max. Energy } \\
\text { Consumed }\end{array}$ & $\begin{array}{c}\text { Min. Energy } \\
\text { Consumed }\end{array}$ & $\begin{array}{c}\text { Max. Energy } \\
\text { Consumed }\end{array}$ & $\begin{array}{c}\text { Min. Energy } \\
\text { Consumed }\end{array}$ \\
\hline Raw & 6210 & 1242 & 1872 & 374.4 \\
Lossless & 4146.2 & 835.5 & 5619 & 4618 \\
\hline Lossy & 632.8 & 136.4 & 6667 & 6517 \\
\hline
\end{tabular}

\section{PLATFORM}

We have chosen two platforms for evaluating the proposed idea. The first chosen platform is PXA27x processor is an integrated microprocessor which has dynamic voltage and frequency scaling. The available frequencies are in the range from 13 to $416 \mathrm{MHz}$. The tables III and IV shows the operating points and corresponding power consumption that is possible in the PXA27x processors [2]. We take these operating points in order to find out an optimal frequency for the processor that is involved in mild or higher processing activity. This will help in finding out the optimal operating point for a processor in an offline manner.

The second platform chosen for this purpose is OMAP3530 (beagleboard) and table V provides measures of the OMAP 3530 processor power consumption at different operating frequencies. It is taken from [5]. Also [4] gives the following observation about the beagleboard. Typically,

- If the bit rate is less than $2 \mathrm{~KB} / \mathrm{sec}$, CPU should run at maximum $125 \mathrm{MHz}$.

- If the bit rate is less than $4 \mathrm{~KB} / \mathrm{sec}, \mathrm{CPU}$ should run at maximum $250 \mathrm{MHz}$.

- If the bit rate is less than $8 \mathrm{~KB} / \mathrm{sec}, \mathrm{CPU}$ should run at maximum $500 \mathrm{MHz}$.

\section{ROUTING PROPOSAL}

The network architecture consists of different nodes that can operate at different frequency levels. If a node operates at the higher frequency then it will be able to process the task fastly, consume more energy and this will be helpful in determining the optimal operating frequency. 
International Journal of Ad hoc, Sensor \& Ubiquitous Computing (IJASUC) Vol.3, No.4, August 2012

The problem is modelled in subsection 4.1 and the routing solution is proposed in the subsections 4.2 and 4.3 for PXA27x and Beagleboard respectively.

\subsection{Model}

The network consists of $\mathrm{N}$ nodes $\mathrm{n}=1,2, \ldots, \mathrm{N}$ and each node can be operated on $\mathrm{M}$ discrete frequency levels $\mathrm{m}=1,2, \ldots, \mathrm{M}$. The processor activity level is shown with the help of a variable $\mathrm{a}=1,2, \ldots, \mathrm{A}$. We have to map this processor activity level to a certain frequency level $\mathrm{m}$ so that the node $\mathrm{n}$ can operate on that frequency. This will help in determining the optimal processing frequency for a certain processor that meets the data reporting deadline and also helps in optimizing the overall network energy consumption. The processor activity level depends whether this node is located close to the sink or not. In that case, it will have a large processor activity otherwise it will have a low processor activity level. This will help in determining the voltage on which we want the device to operate.

\subsection{Frequency Selection for PXA27x}

The mapping function will map the node to a certain frequency in an offline manner. The processor activity level is determined with the help of the position of node with respect to the sink. The node which is located near the sink has the higher activity level as it has to gather the packets that have been generated from the far-off node. Thus, we choose:

a $\alpha$ ( 1 Distance of node from the sink)

Where, the sink is the node in the wireless sensor network which is responsible for collecting all the data. Thus, we choose:

$$
\mathrm{h}=\text { Number of hops from the sink }
$$

In order to choose a frequency for the processor, we map the variable a to the frequency such that,

$$
\mathrm{f} \alpha(1 / \mathrm{h})
$$

And we try to choose the minimal frequency as well to minimize the energy consumption. As we have selected various operating points and each point has specific power consumption. We choose the frequency of a processor based on its activity level, a.

\subsection{Frequency Selection for Beagleboard}

The mapping function for beagle board depends on the processor activity which depends on the data rate that can be supported. For a certain processing activity, we can use the supported data rate listed in the previous section. This gives the maximum frequency that can be assigned to a node w.r.t its distance from the sink (in number of hops) from the sink. If distance from sink is larger, a lower frequency will be selected and higher frequency will be chosen for a node located near to the sink. 
International Journal of Ad hoc, Sensor \& Ubiquitous Computing (IJASUC) Vol.3, No.4, August 2012

\section{EXPERIMENTAL ANALYSIS}

We choose the network shown in figure 2 for evaluation and the simulation parameters are described in table 3. The ideal MAC is considered in this case and we use a $\mathrm{C}++$ based simulator to perform the experiments. The proposed DVFS approaches are implemented on the chosen multimedia wireless sensor nodes. The experiments and the results are described in the following sub-sections.

Figure 2. Simulated Network

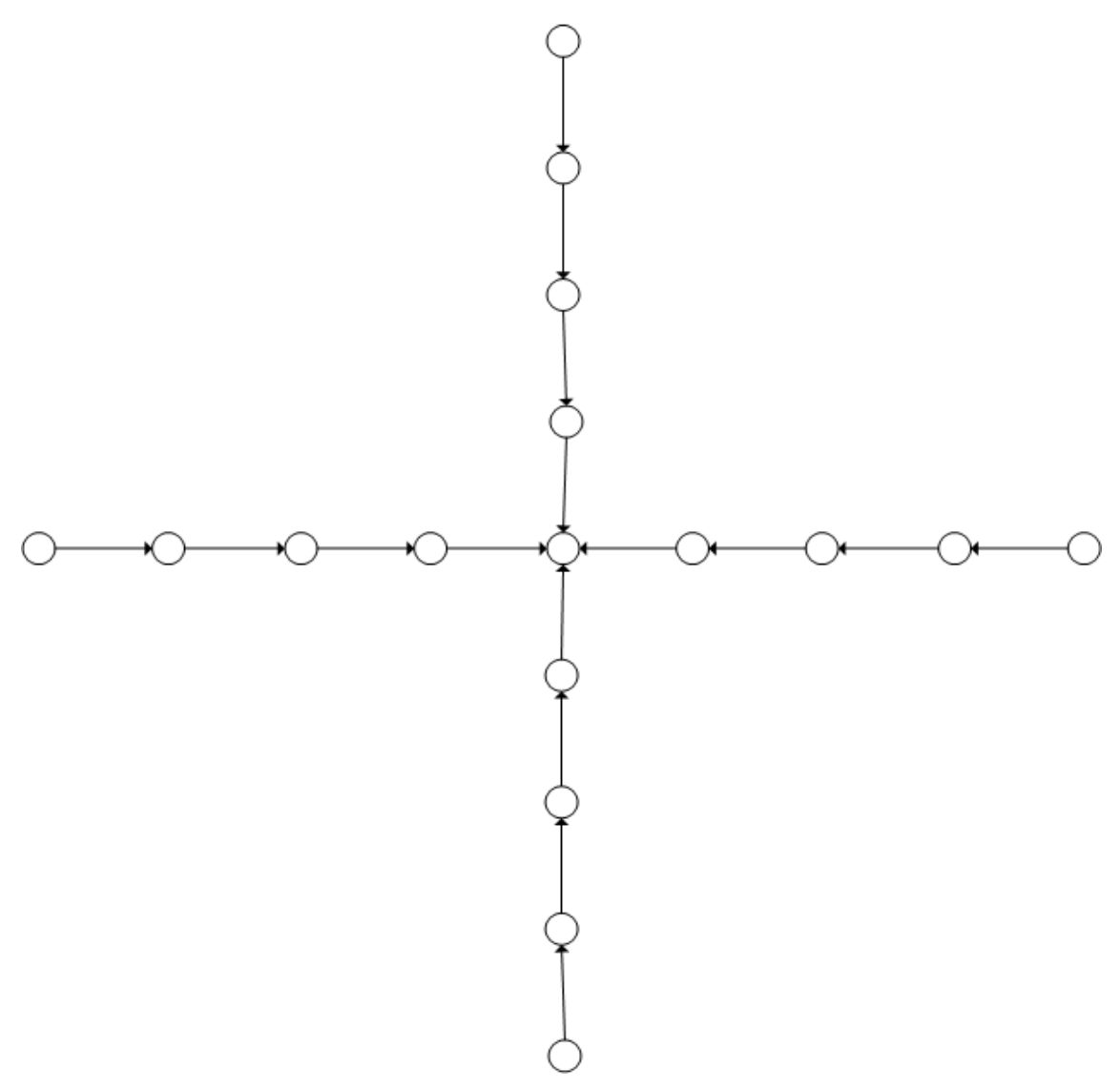

Table 3. Simulation Parameters.

\begin{tabular}{|c|c|}
\hline Parameter & Value \\
\hline Network size & 17 \\
\hline Routing Technique & Geographical \\
\hline Traffic Generation Model & Constant Bit Rate \\
\hline
\end{tabular}


International Journal of Ad hoc, Sensor \& Ubiquitous Computing (IJASUC) Vol.3, No.4, August 2012

\subsection{PXA27x}

The properties for PXA27x are shown in tables 4 and 5. We use these properties to select the frequency based on the frequency selection function described in section 4.2. If we consider three processing frequencies such as 125,250 and $500 \mathrm{MHz}$ for sending the data from source to sink, then the processing results are described in table 6 considering that the transmission rate is not a bottleneck to the transmission rate. These results show that for processing an application, we can use the proposed design approach for data reporting. As its end-to-end delay lies between the approaches where either all the nodes are operating at the minimum or the maximum frequencies. Choosing lower frequency will decrease the processor power consumption but it will increase the end-to-end packet reporting delay where each application will take 5 micro seconds to process on the processor for the lowest frequency. Choosing maximum frequency for all the nodes increases the power consumption but decreases the endto-end delay.

Table 4. Clock Frequencies for the PXA27X processor [2].

\begin{tabular}{|c|c|c|c|c|c|}
\hline OP & Core (f) & Bus (f) & Memory (f) & SDRAM (f) & Core(f) \\
\hline $\mathbf{1}$ & 13 & 13 & 13 & 13 & $850 \mathrm{mV}$ \\
$\mathbf{2}$ & 104 & 104 & 104 & 104 & $900 \mathrm{mV}$ \\
\hline $\mathbf{3}$ & 208 & 208 & 208 & 104 & $1125 \mathrm{mV}$ \\
\hline $\mathbf{4}$ & 312 & 208 & 208 & 104 & $1200 \mathrm{mV}$ \\
\hline $\mathbf{5}$ & 416 & 208 & 208 & 104 & $1250 \mathrm{mV}$ \\
\hline
\end{tabular}

Table 5. Operating Point Characterization for the PXA27X processor [2].

\begin{tabular}{|c|c|c|c|c|}
\hline & \multicolumn{2}{|c|}{ cpu-bench } & \multicolumn{2}{c|}{ mem-bench } \\
\hline OP & Power $(\mathbf{m W})$ & T(sec) & Power(mW) & T(sec) \\
\hline $\mathbf{5}$ & 568 & 2.21 & 616 & 0.96 \\
\hline $\mathbf{4}$ & 432 & 2.95 & 472 & 1.18 \\
\hline $\mathbf{3}$ & 304 & 4.41 & 336 & 1.65 \\
\hline $\mathbf{2}$ & 172 & 8.83 & 188 & 3.18 \\
$\mathbf{1}$ & 108 & 74 & 112 & 25.37 \\
\hline
\end{tabular}

Table 6. Experimental Results for PXA 27x.

\begin{tabular}{|c|c|c|c|}
\hline & $\begin{array}{c}\text { Max. Power } \\
\text { Consumed }\end{array}$ & $\begin{array}{c}\text { Min. Power } \\
\text { Consumed }\end{array}$ & End-to-end Delay \\
\hline Proposed Approach & $303 \mathrm{~mW}$ & $228 \mathrm{mw}$ & 13.75 micro sec. \\
\hline Min. Energy & $228 \mathrm{~mW}$ & $228 \mathrm{~mW}$ & 20 micro sec. \\
Max. Energy & $303 \mathrm{~mW}$ & $303 \mathrm{~mW}$ & 5 micro sec. \\
\hline
\end{tabular}




\subsection{Beagleboard}

The power consumption versus frequency for a particular application executing on Beagleboard is listed in table 7. The frequency for this board is selected based on the processor activity level described in section 4.3. Here, we know the data rate that can be achieved for a particular frequency and we use this value to calculate the bottleneck data rate supported. This is shown in table 8 where we can select the processor frequency to minimize the processor power consumption by increasing the data rate. The proposed approach for frequency selection and the maximum frequency selection approach have the best data rate at the network bottleneck and the energy consumption of the proposed approach is comparable to the minimum frequency selection approach.

Table 7. Power Consumption versus Frequency for Beagleboard [5].

\begin{tabular}{|c|c|}
\hline Frequency $(\mathbf{M H z})$ & $\mathbf{P}(\mathbf{m W})$ \\
\hline 125 & 57 \\
\hline 250 & 130 \\
\hline 500 & 303 \\
\hline 550 & 371 \\
\hline 600 & 445 \\
\hline
\end{tabular}

Table 8. Experimental Results for Beagleboard.

\begin{tabular}{|c|c|c|c|}
\hline & $\begin{array}{c}\text { Max. Power } \\
\text { Consumed }\end{array}$ & $\begin{array}{c}\text { Min. Power } \\
\text { Consumed }\end{array}$ & $\begin{array}{c}\text { Data Rate at } \\
\text { Network Bottleneck }\end{array}$ \\
\hline Proposed Approach & $303 \mathrm{~mW}$ & $228 \mathrm{mw}$ & $8 \mathrm{~KB} / \mathrm{sec}$ \\
Min. Energy & $228 \mathrm{~mW}$ & $228 \mathrm{~mW}$ & $2 \mathrm{~KB} / \mathrm{sec}$ \\
\hline Max. Energy & $303 \mathrm{~mW}$ & $303 \mathrm{~mW}$ & $8 \mathrm{~KB} / \mathrm{sec}$ \\
\hline
\end{tabular}

\section{Conclusions}

In this article, we have coupled various node level optimizations with the global optimizations to help in further optimizing the global power consumption in the wireless sensor networks. The local node level optimizations that have been considered include data compression and DVFS and these techniques were coupled and compared with the routing energy consumption for different network scenarios. The results show that we can achieve better end-to-end data reporting delay and minimize the overall network energy consumption by considering the node level optimizations while making a routing decision.

\section{REFERENCES}

[1] L. Nachman, J. Huang, J. Shahabdeen, R. Adler \& R. Kling, (2008) "IMOTE2: Serious Computation at the Edge", Proceedings of International Wireless Communications and Mobile Computing Conference, Crete Island.

[2] A. Castagnetti, C. Belleudy, S. Bilavarn \& M. Auguin, (2010) "Power Consumption Modeling for DVFS Exploitation", Proceedings of 13th Euromicro Conference on Digital System Design: Architectures, Methods and Tools, Lille. 
International Journal of Ad hoc, Sensor \& Ubiquitous Computing (IJASUC) Vol.3, No.4, August 2012

[3] A. Ravinagarajan, D. Dondi \& T.S. Rosing, (2010) "DVFS based task scheduling in a harvesting WSN for Structural Health Monitoring", Proceedings of Design, Automation and Test in Europe Conference and Exhibition (DATE), Dresden.

[4] S. Bilavarn, A. Castagnetti \& L. Rodrigue, (2011) “A Video Monitoring Application for Wireless Sensor Networks using IEEE 802.15.4”, Proceedings of ARCS, Como.

[5] J. Kriegel, F. Broekaert, A. Pegatoquet \& M. Auguin, (2010) "Power optimization technique applied to real-time video application", 13th Sophia Antipolis Microelectronics Forum (SAME), University Booth, Valbonne, France. 